\title{
Can diffusion-weighted imaging predict tumor grade and expression of Ki-67 in breast cancer? A multicenter analysis
}

\author{
Alexey Surov ${ }^{1 *}$ DD, Paola Clauser ${ }^{2}$, Yun-Woo Chang $^{3}$, Lihua Li ${ }^{4}$, Laura Martincich ${ }^{5}$, Savannah C. Partridge ${ }^{6}$, \\ Jin You Kim ${ }^{7}$, Hans Jonas Meyer ${ }^{1}$ and Andreas Wienke ${ }^{8}$
}

\begin{abstract}
Background: Numerous studies have analyzed associations between apparent diffusion coefficient (ADC) and histopathological features such as Ki-67 proliferation index in breast cancer (BC), with mixed results. The purpose of this study was to perform a multicenter analysis to determine relationships between ADC and expression of Ki-67 and tumor grade in BC.

Methods: For this study, data from six centers were acquired. The sample comprises 870 patients (all female; mean age, $52.6 \pm 10.8$ years). In every case, breast magnetic resonance imaging with diffusion-weighted imaging was performed. The comparison of ADC values in groups was performed by Mann-Whitney $U$ test where the $p$ values are adjusted for multiple testing (Bonferroni correction). The association between ADC and Ki-67 values was calculated by Spearman's rank correlation coefficient. Sensitivity, specificity, negative and positive predictive values, accuracy, and AUC were calculated for the diagnostic procedures. ADC thresholds were chosen to maximize the Youden index.

Results: Overall, data of 870 patients were acquired for this study. The mean ADC value of the tumors was $0.98 \pm 0.22 \times$ $10^{-3} \mathrm{~mm}^{2} \mathrm{~s}^{-1}$. ROC analysis showed that it is impossible to differentiate high/moderate grade tumors from grade 1 lesions using ADC values. Youden index identified a threshold ADC value of 1.03 with a sensitivity of $56.2 \%$ and specificity of $67.9 \%$. The positive predictive value was $18.2 \%$, and the negative predictive value was $92.4 \%$. The level of the Ki-67 proliferation index was available for 845 patients. The mean value was $12.33 \pm 21.77 \%$. ADC correlated with weak statistical significant with expression of $\mathrm{Ki}-67(p=-0.202, p<0.001)$. ROC analysis was performed to distinguish tumors with high proliferative potential from tumors with low expression of Ki-67 using ADC values. Youden index identified a threshold ADC value of 0.91 (sensitivity 64\%, specificity 50\%, positive predictive value $67.7 \%$, negative predictive value $45.0 \%$ ).
\end{abstract}

Conclusions: ADC cannot be used as a surrogate marker for proliferation activity and/or for tumor grade in breast cancer.

Keywords: Breast cancer, ADC, DWI, Ki-67

\section{Background}

Breast cancer $(\mathrm{BC})$ is the most common noncutaneous malignancy among women, representing four in ten female cancer survivors in the United States [1]. Different imaging modalities such as mammography, ultrasound, and magnetic resonance imaging (MRI) play an essential role in the diagnosis and local staging of $\mathrm{BC}$. According to the

\footnotetext{
*Correspondence: alexey.surov@medizin.uni-leipzig.de

${ }^{1}$ Department of Diagnostic and Interventional Radiology, University of

Leipzig, Liebigstrasse 20, 04103 Leipzig, Germany

Full list of author information is available at the end of the article
}

literature, imaging not only can document breast lesions but also can predict histopathological features of $\mathrm{BC}[2-5]$. For instance, Seo et al. reported that the human epidermal growth factor receptor 2 (HER2)-positive subtype of BC was associated with a higher BI-RADS (Breast Imaging Reporting and Data System) category [2]. Some authors also indicated that several imaging features can provide information about proliferation potential or expression of Ki-67 in BC [5]. Szabo et al. reported that rim enhancement on dynamic MRI was associated with high expression of $\mathrm{Ki}-67$ and poor prognosis of $\mathrm{BC}$ [5]. Furthermore,

(C) The Author(s). 2018 Open Access This article is distributed under the terms of the Creative Commons Attribution 4.0 International License (http://creativecommons.org/licenses/by/4.0/), which permits unrestricted use, distribution, and 
numerous studies analyzed associations between diffusion-weighted imaging (DWI) and histopathological features in $\mathrm{BC}$, including associations between apparent diffusion coefficient (ADC) and expression of Ki-67 [4, 6, 7]. However, the reported data were mixed. Whereas some authors found significant correlations between ADC and Ki-67 in BC, other did not [8-13]. For example, Li et al. observed a moderate statistically significant correlation between ADC and Ki-67 ( $r=-0.566, p=0.025)$ [8]. Similar results were also reported by Mori et al. [9]. These authors suggested that ADC would be practical to use for estimating the Ki-67 labeling index [9]. However, Aydin et al. could find only a weak negative correlation between ADC and Ki-67-values in $\mathrm{BC}(r=-0.279 ; p=0.029)$ [10]. Finally, some authors did not identify statistically significant correlations between these parameters [11-13]. Similarly, data about relationships between tumor grade and ADC were also inconsistent. These facts question the possibility to use $\mathrm{ADC}$ as a surrogate marker for proliferation activity in $\mathrm{BC}$ in clinical practice. The purpose of the present study was to provide evidence-based data regarding associations between ADC and expression of Ki-67 as well tumor grade in $\mathrm{BC}$.

\section{Methods}

\section{Data acquisition and patients}

For this study, the MEDLINE library was screened for associations between ADC and Ki-67 in BC up to December 2017. The following search words were used: "DWI or diffusion weighted imaging or diffusion-weighted imaging or ADC or apparent diffusion coefficient AND Ki-67 OR KI67 OR ki67 OR ki-67 OR mitotic index OR proliferation index OR MIB 1 OR MIB-1 OR mitosis index AND breast cancer OR breast carcinoma." Overall, 41 items were identified.

In the next step, corresponding authors of all identified reports were contacted via email with a request to provide the data of the investigated patients, including the following for every case: age, precise histopathological diagnosis, tumor grade, mean ADC values, and Ki-67 index. Overall, six of them provided their data [14-19]. Data were provided from the following centers:

- Department of Medical and Biological Sciences, Institute of Diagnostic Radiology, Azienda Ospedaliero Universitaria Santa Maria della Misericordia, University of Udine, Udine, Italy (center 1)

- Department of Radiology, Soon Chun Hyang University Hospital, Seoul, Republic of Korea (center 2)

- Institute of Biomedical Engineering and Instrumentation, Hangzhou Dianzi University, Hangzhou, China (center 3)

- Unit of Radiology, Cancer Institute, Institute for Cancer Research and Treatment of Candiolo (IRCC), Turin, Italy (center 4)
- Department of Radiology, University of Washington, Seattle, WA, USA (center 5)

- Department of Radiology, Pusan National University Hospital, Pusan National University School of Medicine and Medical Research Institute, Busan, Republic of Korea (center 6)

The acquired sample comprises 870 patients (all female; mean age, $52.6 \pm 10.8$ years; median age, 52 years; range, 24-85 years). The patients had a variety of breast tumor histologic types (Table 1 ). In every case, breast MRI with DWI was performed with a clinical scanner (1.5 and 3.0 T) with dedicated breast radiofrequency coils. MRI equipment and imaging protocols varied across centers (Table 2).

\section{Statistical analysis}

Continuous variables were described by mean value, median value, and SD. Categorical variables were given as relative frequencies. The comparison of $\mathrm{ADC}$ values in groups was performed by Mann-Whitney $U$ tests where the $p$ values are adjusted for multiple testing (Bonferroni correction). The association between ADC and Ki-67 values was calculated by Spearman's rank correlation coefficient. Sensitivity, specificity, negative and positive predictive values, accuracy, and AUC were calculated for the diagnostic procedures. ADC thresholds were chosen to maximize the Youden index.

\section{Results}

\section{ADC values and tumor grade/subtypes}

Overall, data of 870 patients were acquired for this study. The majority of tumors were invasive ductal carcinoma (IDC; $81.95 \%$ ), with a limited number of other subtypes (Table 1$)$. The mean ADC value $\left(\times 10^{-3} \mathrm{~mm}^{2} \mathrm{~s}^{-1}\right)$ of the tumors was $0.98 \pm 0.22$; the median value was 0.95 ; and the range was $0.41-2.18$. Ductal carcinoma in situ (DCIS) showed statistically significant higher ADC values $\left(1.11 \pm 0.24 \times 10^{-3} \mathrm{~mm}^{2} \mathrm{~s}^{-1}\right)$ than IDC $(0.97 \pm 0.21 \times$ $\left.10^{-3} \mathrm{~mm}^{2} \mathrm{~s}^{-1} ; P=0.001\right)$ and invasive lobular carcinoma

Table 1 Analyzed tumors

\begin{tabular}{lll}
\hline Diagnosis & No. & $\%$ \\
\hline DCIS & 45 & 5.17 \\
IDC & 713 & 81.95 \\
ILC & 57 & 6.55 \\
Combined IDC/ILC & 9 & 1.03 \\
Mucinous carcinoma & 7 & 0.81 \\
Tubular carcinoma & 3 & 0.35 \\
Metaplastic carcinoma & 2 & 0.23 \\
Unspecified & 34 & 3.91 \\
Total & 870 & 100 \\
\hline
\end{tabular}

Abbreviations: DCIS Ductal carcinoma in situ, IDC Invasive ductal carcinoma. ILC Invasive lobular carcinoma 
Table 2 Patients and magnetic resonance imaging techniques

\begin{tabular}{|c|c|c|c|}
\hline Centers & No. of patients & MRI equipment and field strength & DWI sequences and b-values \\
\hline 1 & 115 & $\begin{array}{l}\text { 1.5-T scanner (MAGNETOM Avanto; Siemens Medical } \\
\text { Systems, Erlangen, Germany) }\end{array}$ & $\begin{array}{l}\text { Single-shot echo-planar sequence (TR/TE: 7100/84 ms); b-values: } \\
0-1000 \mathrm{~s} / \mathrm{mm}^{2}\end{array}$ \\
\hline 2 & 335 & $\begin{array}{l}\text { 1.5-T scanner (Sonata; Siemens Medical Systems, } \\
\text { Erlangen, Germany) }\end{array}$ & $\begin{array}{l}\text { Single-shot echo-planar sequence (TR/TE: 5000/110 ms); b-values } \\
0-1000 \mathrm{~s} / \mathrm{mm}^{2}\end{array}$ \\
\hline 3 & 82 & $\begin{array}{l}\text { 3.0-T scanner (MAGNETOM Verio, Siemens Medical } \\
\text { Systems, Erlangen, Germany) }\end{array}$ & $\begin{array}{l}\text { Single-shot echo-planar sequence (TR/TE: 7000/85 ms); b-values: } \\
50-1000 \mathrm{~s} / \mathrm{mm}^{2}\end{array}$ \\
\hline 4 & 143 & $\begin{array}{l}\text { 1.5-T scanner (GE Healthcare Life Sciences, Milwaukee, } \\
\text { WI, USA) }\end{array}$ & $\begin{array}{l}\text { Single-shot echo-planar sequence (TR/TE: 7000/85 ms); b-values: } \\
0-900 \mathrm{~s} / \mathrm{mm}^{2}\end{array}$ \\
\hline 5 & 107 & $\begin{array}{l}\text { 3-T scanner (Achieva TX; Philips Healthcare, Best, The } \\
\text { Netherlands) }\end{array}$ & $\begin{array}{l}\text { Single-shot echo-planar sequence (TR/TE: 5336/ } 61 \mathrm{~ms}) \text {; b-values: } \\
0-800 \mathrm{~s} / \mathrm{mm}^{2}\end{array}$ \\
\hline 6 & 88 & $\begin{array}{l}\text { 3-T scanner (Trio Tim; Siemens Medical Systems, } \\
\text { Erlangen, Germany) }\end{array}$ & $\begin{array}{l}\text { Single-shot echo-planar sequence (TR/TE: } 6600 / 91 \mathrm{~ms} \text { ); b-values: } \\
0-1000 \mathrm{~s} / \mathrm{mm}^{2}\end{array}$ \\
\hline
\end{tabular}

Abbreviations: MRI Magnetic resonance imaging, DWI Diffusion-weighted imaging, TR Repetition time, TE Echo time

(ILC; $\left.1.01 \pm 0.21 \times 10^{-3} \mathrm{~mm}^{2} \mathrm{~s}^{-1} ; P=0.044\right)$. There were no significant differences in ADC values between IDC and ILC (Fig. 1). Furthermore, ADC values differed between tumor grades. Grade 1 tumors had significantly higher ADC values $\left(1.09 \pm 0.27 \times 10^{-3} \mathrm{~mm}^{2} \mathrm{~s}^{-1}\right)$ than grade $2\left(0.97 \pm 0.21 \times 10^{-3} \mathrm{~mm}^{2} \mathrm{~s}^{-1}, P<0.001\right)$ and grade 3 $\left(0.95 \pm 0.21 \times 10^{-3} \mathrm{~mm}^{2} \mathrm{~s}^{-1}, \quad P<0.001\right)$ lesions. No significant differences in ADC values were observed between grades 2 and 3 tumors $(P=1.00$ ) (Fig. 2).

Next, ROC analysis was performed to differentiate high/moderate grade tumors from grade 1 lesions using ADC values. Youden index identified a threshold ADC value of 1.03 with a sensitivity of $56.2 \%$ and specificity of $67.9 \%$. The positive predictive value was $18.2 \%$, and the negative predictive value was $92.4 \%$. ROC analysis (Fig. 3) showed that the AUC was 0.657 .

\section{ADC values and $\mathrm{Ki}-67$ level}

The level of the Ki-67 proliferation index was available for 845 patients. The mean value was $12.33 \pm$ $21.77 \%$; the median value was $30 \%$; and the range was $1-100 \%$. ADC correlated weakly with expression of Ki-67 $(p=-0.202, P<0.001)$ (Fig. 4). Furthermore, ADC correlated with Ki-67 in the IDC subgroup $(p=-0.173, P<0.001)$ and the ILC subgroup $(p=-0.296, P=0.037)$, but not in the DCIS subgroup $(p=0.027, P=0.859)$.
A Ki-67 value of $25 \%$ was used as the threshold for discriminating between tumors with low Ki-67 expression $(<25 \%)$ and high Ki-67 expression ( $\geq 25 \%)$. Tumors with low expression of Ki-67 $\quad(n=528$ [62.49\%]) had higher ADC values than tumors with high expression of Ki-67 $(n=317$ [37.51\%]) (0.99 \pm $0.22 \times 10^{-3} \mathrm{~mm}^{2} \mathrm{~s}^{-1}$ vs $0.95 \pm 0.21 \times 10^{-3} \mathrm{~mm}^{2} \mathrm{~s}^{-1}$, respectively; $P=0.005)$. However, $\mathrm{ADC}$ values of the subgroups overlapped significantly (Fig. 5).

In the next step, ROC analysis was performed to distinguish tumors with high proliferative potential from tumors with low expression of Ki-67 using ADC values. Youden index identified a threshold ADC value of 0.91 . Using this threshold resulted in sensitivity of $64 \%$ and specificity of $50 \%$. The positive predictive value was $67.7 \%$, the negative predictive value was $45.0 \%$, and the AUC was 0,574 (Fig. 6). Other threshold values of Ki-67 were also analyzed (see Table 3 ).

\section{Discussion}

This is the first multicenter study about relationships between ADC and histopathological features such as expression of Ki-67 and tumor grade in BC. Overall, it addresses a key question of whether imaging parameters can reflect clinically relevant histopathological findings. If so, then imaging, in particular ADC values, can be used as surrogate markers for tumor biology in BC.

Table 3 Results of ROC analysis

\begin{tabular}{lllllll}
\hline Ki-67 expression threshold & ADC threshold & Sensitivity & Specificity & AUC & Positive predictive value & Negative predictive value \\
\hline$\geq 10 \%$ & 0.951 & 0.704 & 0.542 & 0.644 & 0.267 & 0.885 \\
$\geq 20 \%$ & 0.913 & 0.700 & 0.494 & 0.613 & 0.498 & 0.696 \\
$\geq 30 \%$ & 0.913 & 0.639 & 0.512 & 0.590 & 0.712 & 0.430 \\
$\geq 40 \%$ & 0.821 & 0.808 & 0.366 & 0.576 & 0.844 & 0.892 \\
$\geq 50 \%$ & 0.821 & 0.807 & 0.413 & 0.600 & 0.263 \\
\hline
\end{tabular}




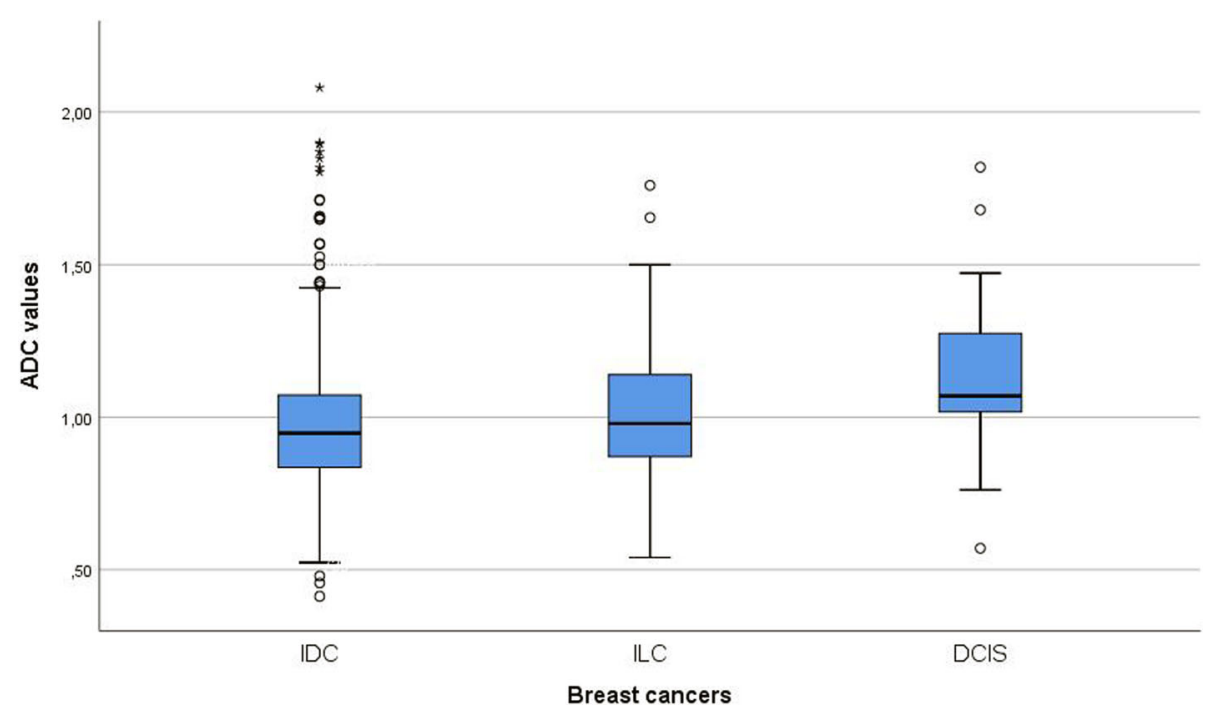

Fig. 1 Comparison of apparent diffusion coefficient (ADC) values between ductal carcinoma in situ (DCIS), invasive ductal carcinoma (IDC) and invasive lobular carcinoma (ILC)

Ki-67 is a well-established biomarker in BC [20, 21]. According to the literature, Ki-67 before and after neoadjuvant chemotherapy can predict the prognosis for patients with BC [21]. Furthermore, pretherapeutic Ki-67 is associated with pathological complete response after neoadjuvant chemotherapy in patients with BC [22]. In addition, Ki-67 is associated with overall and disease-free survival of patients with $\mathrm{BC}$ [23]. Therefore, it can be important in clinical practice to predict expression of Ki-67 on the basis of imaging.

ADC reflects diffusion of water molecules in tissue [24, 25]. Recently, a meta-analysis identified inverse correlations between ADC and cell count in several malignant and benign tumors [25]. Furthermore, it has been shown that ADC was associated with expression of Ki-67 in different lesions [26]. Several mechanisms may explain this association. Ki-67 is a nonhistone nuclear protein synthesized throughout the whole cell cycle except the $G_{0}$ phase $[27,28]$. Cytoplasmic proteins and cytoplasmic viscosity increase during mitosis [29]. These factors may influence water diffusion and ADC. Additionally, water diffusion may be affected by intracellular mitotic membranes.

As mentioned above, numerous prior studies investigated the role of $\mathrm{ADC}$ values in $\mathrm{BC}$ diagnosis and treatment. However, the reported results regarding associations between $\mathrm{ADC}$ and histopathology were

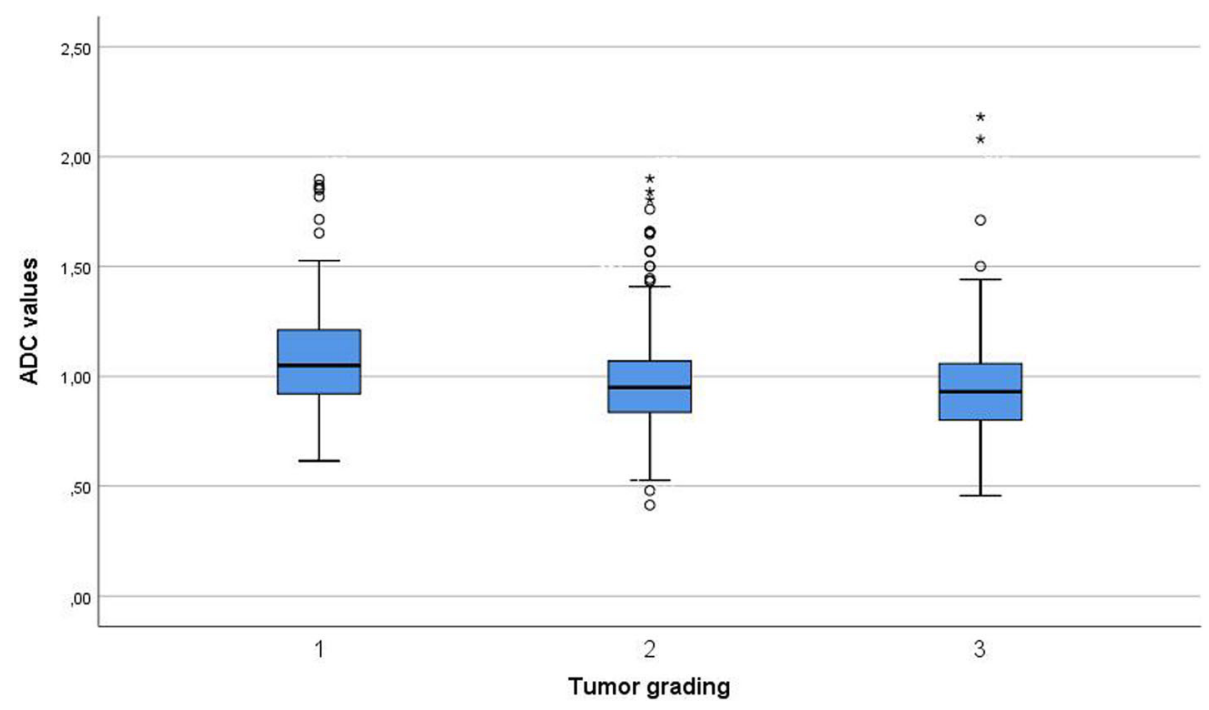

Fig. 2 Comparison of apparent diffusion coefficient (ADC) values between grades 1, 2, and 3 tumors 


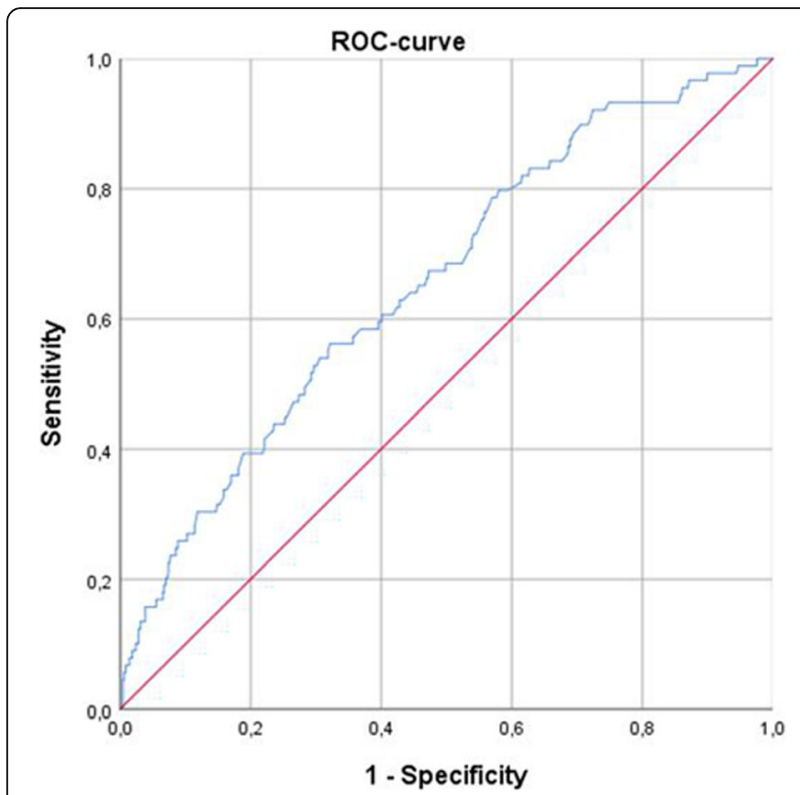

Fig. 3 ROC curve for use of apparent diffusion coefficient (ADC) values in distinguishing grades 2 and 3 breast cancer from grade 1 tumors. Threshold $A D C$ value $=1.03$, sensitivity $=56.2 \%$, specificity $=67.9 \%$, positive predictive value $=18.2 \%$, and negative predictive value $=92.4 \%$

inconclusive. Interpretation of prior results is complicated by differences in study design and analysis. The published radiological studies used different values of Ki-67 expression to discriminate tumors with low and high proliferative activity. For example, in the study of Zhuang et al., a Ki-67 level of $\geq 14 \%$ was considered to indicate high proliferation, and $<14 \%$ was considered to indicate low proliferation [30]. Some other studies used a threshold value of $20 \%[15,16]$ or defined more than two Ki-67 categories. For example, De Felice et al. categorized Ki-67 expression as follows: low Ki-67 ( $\leq 14 \%)$, intermediate Ki-67 (15-30\%), and high Ki-67 ( $\geq 30 \%)$ [13]. According to the meta-analysis of Petrelli et al., based on data of 64,196 patients, a Ki-67 cutoff $>25 \%$ is associated with a greater risk of death than lower expression rates [31]. Therefore, a reevaluation of the previous studies on associations between ADC and Ki-67 expression was needed.

The present study suggests that ADC cannot be used as a surrogate marker for proliferation activity in BC. In fact, although ADC values between tumors with high expression of Ki-67 ( $\geq 25 \%)$ differed from those with low levels of Ki-67 $(<25 \%)$, the calculated specificity and sensitivity were too low. This applied also for several alternative thresholds of Ki-67 expression ranging from $10 \%$ to $50 \%$. Similar results were also observed for distinguishing low-grade and intermediate/high-grade tumors. Statistical analysis identified that grade 1 lesions had higher ADC values than grades 2 and 3 tumors. However, the ROC analysis showed that a possible use of $\mathrm{ADC}$ for discrimination of tumor grade in $\mathrm{BC}$ has very low specificity and sensitivity. Furthermore, we found that DCIS had statistically significant higher ADC values than IDC and ILC. However, ADC values also overlapped also overlapped distinctly in these subtypes. Therefore, use of ADC does not provide specific information regarding tumor biology in $\mathrm{BC}$ that can be used reliably in clinical practice.

The present study is the largest to date on this topic, to our knowledge. However, it has some limitations. The involved patients were investigated with use of different MRI equipment with different technical parameters, such as field strength, DWI sequences, and b-values. This may broaden the range of ADC values in the study and may have influenced

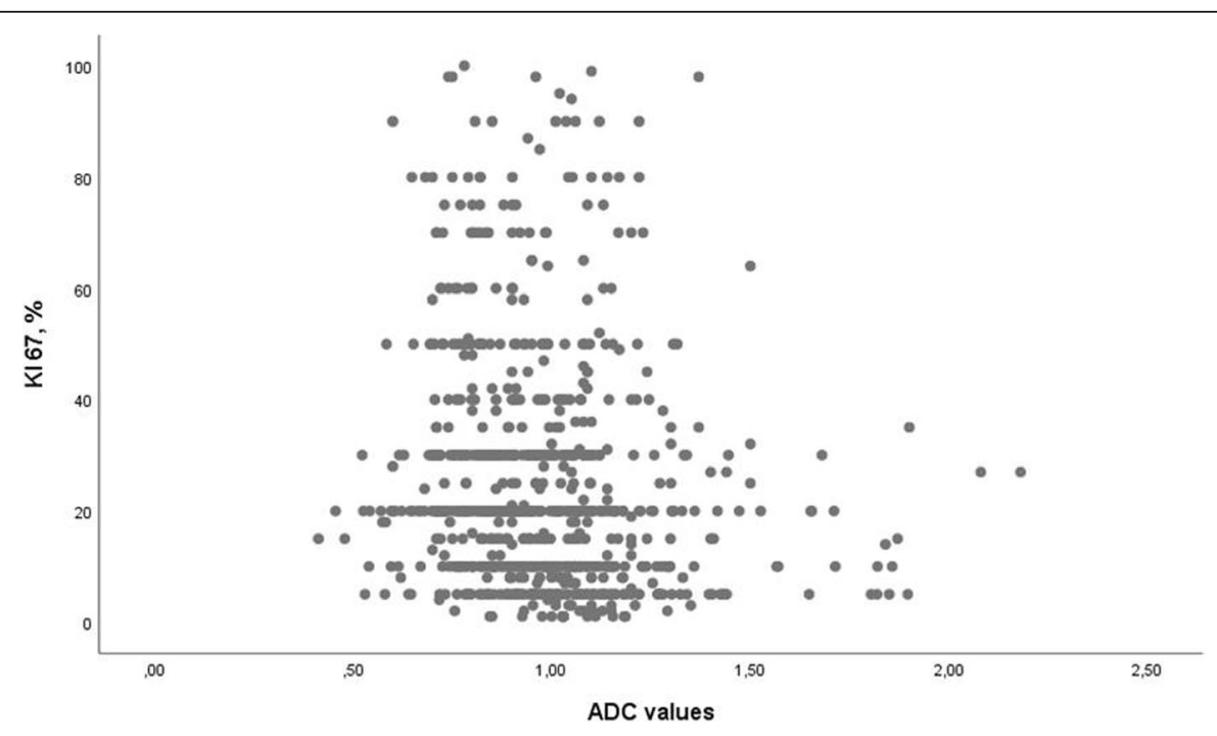

Fig. 4 Correlation between apparent diffusion coefficient (ADC) and expression of Ki-67. The calculated correlation coefficient was $-0.202(P<0.001)$ 


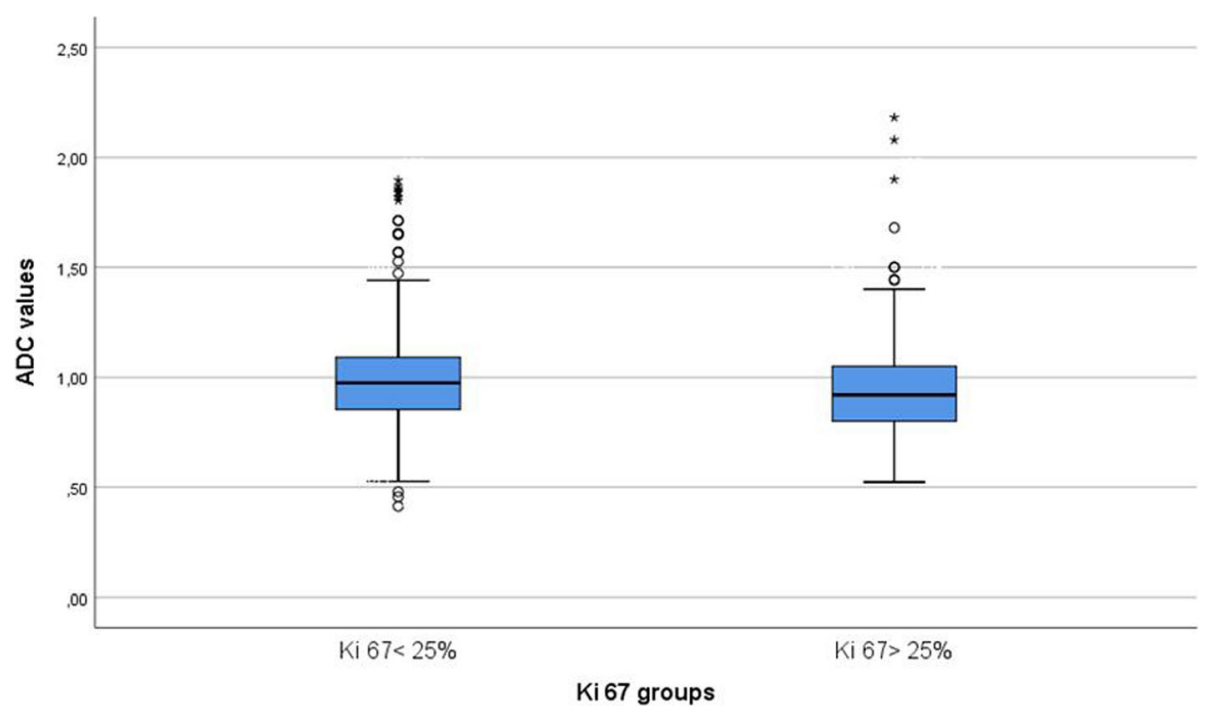

Fig. 5 Comparison of apparent diffusion coefficient (ADC) values between tumors with low expression of Ki-67 $(<25 \%)$ and high expression of Ki-67 ( $\geq 25 \%)$

our results. Furthermore, the patient samples consisted predominantly of only three tumor subgroups, namely DCIS, IDC, and ILC. Our study identified that associations between ADC and Ki-67 were different in several subtypes of BC. Moreover, the calculated correlation coefficients for IDC, DCIS, and ILC in our study differed significantly from those for mucinous carcinoma $(r=0.035, p=0.892)$ reported by Onishi et al. [32]. Presumably, associations between

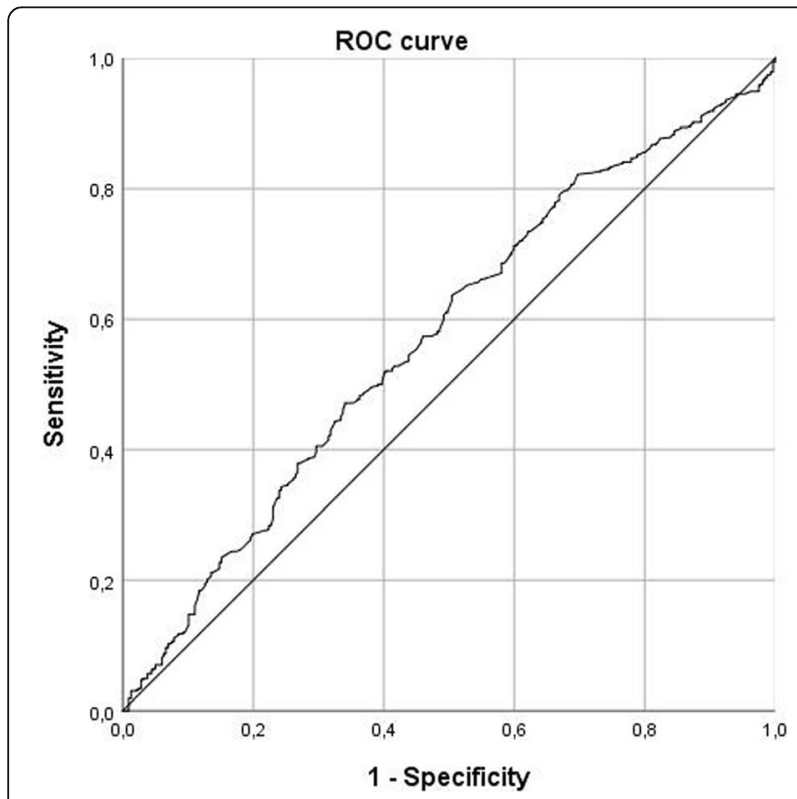

Fig. 6 ROC curve for use of apparent diffusion coefficient (ADC) values in discrimination between tumors with low Ki-67 expression $(<25 \%)$ and high Ki-67 expression ( $\geq 25 \%)$. The threshold ADC value was 0.91 . Sensitivity $=64.4 \%$, specificity $=50.0 \%$
ADC and Ki-67 or tumor grade may be different in other subtypes of BC such as tubular or medullary carcinomas. However, the included tumors represent the most frequent subtypes of $\mathrm{BC}$, whereas other carcinomas are rarer. We did not analyze possible associations between $\mathrm{ADC}$ and other clinically relevant biological features in $\mathrm{BC}$, such as hormonal receptor status and/or HER2 status. This interesting aspect is a goal of further studies.

\section{Conclusions}

Our multicenter study shows that ADC cannot be used as a reliable surrogate marker for proliferative activity and/or for tumor grade in $\mathrm{BC}$.

\section{Availability of data and materials}

The data that support the findings of this study are available from the corresponding author, but restrictions apply to the availability of these data, which were used under license for the present study, and so are not publicly available. Data are, however, available from the authors upon reasonable request and with permission of the corresponding author (AS).

\section{Authors' contributions}

AS made substantial contributions to study conception and design, or acquisition of data, or analysis and interpretation of data. HJM, AW, YWC, LL, $L M, S C P, J Y K$ and PC were involved in drafting the manuscript or revising it critically for important intellectual content. HJM, AW, YWC, LL, LM, SCP, JYK and $P C$ gave final approval of the version to be published. Each author should have participated sufficiently in the work to take public responsibility for appropriate portions of the content. AS, HJM, AW, YWC, LL, LM, SCP, JYK and $P C$ agreed to be accountable for all aspects of the work in ensuring that questions related to the accuracy or integrity of any part of the work are appropriately investigated and resolved. All authors read and approved the final manuscript.

Ethics approval and consent to participate Not applicable. 


\section{Competing interests}

The authors declare that they have no competing interests.

\section{Publisher's Note}

Springer Nature remains neutral with regard to jurisdictional claims in published maps and institutional affiliations.

\section{Author details}

'Department of Diagnostic and Interventional Radiology, University of Leipzig, Liebigstrasse 20,04103 Leipzig, Germany. Department of Biomedical Imaging and Image-guided Therapy, Medical University of Vienna, Währinger Gürtel, 18-20 1090 Vienna, Austria. ${ }^{3}$ Department of Radiology, Soonchunhyang University Hospital, 59 Daesakwan-ro, Yongsan-gu, Seoul 140-743, Republic of Korea. ${ }^{4}$ Institute of Biomedical Engineering and Instrumentation, Hangzhou Dianzi University, Hangzhou, China. ${ }^{5}$ Unit of Radiology, Institute for Cancer Research and Treatment of Candiolo (IRCC), Strada Provinciale 142, 10060 Candiolo, Turin, Italy. ${ }^{6}$ Department of Radiology, University of Washington, 825 Eastlake Avenue E, G2-600, Seattle, WA 98109, USA. 7 Department of Radiology, Pusan National University Hospital, Pusan National University School of Medicine and Medical Research Institute, 1-10, Ami-Dong, Seo-gu, Busan 602-739, Korea. ${ }^{8}$ Institute of Medical Epidemiology, Biostatistics, and Informatics, Martin-Luther-University Halle-Wittenberg, Magdeburger Strasse, 06097 Halle, Germany.

\section{Received: 15 March 2018 Accepted: 18 May 2018}

\section{Published online: 19 June 2018}

\section{References}

1. DeSantis $C E$, Lin CC, Mariotto $A B$, Siegel RL, Stein KD, Kramer JL, Alteri $R$, Robbins AS, Jemal A. Cancer treatment and survivorship statistics, 2014. CA Cancer J Clin. 2014;64(4):252-71.

2. Seo BK, Pisano ED, Kuzimak CM, Koomen M, Pavic D, Lee Y, Cole EB, Lee J. Correlation of HER-2/neu overexpression with mammography and age distribution in primary breast carcinomas. Acad Radiol. 2006;13(10):1211-8.

3. Bae MS, Moon WK, Chang JM, Cho N, Park SY, Won JK, Jeon YK, Moon HG, Han W. Park IA. Mammographic features of calcifications in DCIS: correlation with oestrogen receptor and human epidermal growth factor receptor 2 status. Eur Radiol. 2013;23(8):2072-8.

4. Youk JH, Son EJ, Chung J, Kim JA, Kim EK. Triple-negative invasive breast cancer on dynamic contrast-enhanced and diffusion-weighted MR imaging: comparison with other breast cancer subtypes. Eur Radiol. 2012;22(8):1724-34.

5. Szabo BK, Aspelin P, Kristoffersen Wiberg M, Tot T, Bone B. Invasive breast cancer: correlation of dynamic MR features with prognostic factors. Eur Radiol. 2003;13(11):2425-35.

6. Suo S, Cheng F, Cao M, Kang J, Wang M, Hua J, Hua X, Li L, Lu Q, Liu J, Xu J. Multiparametric diffusion-weighted imaging in breast lesions: association with pathologic diagnosis and prognostic factors. J Magn Reson Imaging. 2017:46(3):740-50

7. Jeh SK, Kim SH, Kim HS, Kang BJ, Jeong SH, Yim HW, Song BJ. Correlation of the apparent diffusion coefficient value and dynamic magnetic resonance imaging findings with prognostic factors in invasive ductal carcinoma. J Magn Reson Imaging. 2011;33(1):102-9.

8. Li L, Wang K, Sun X, Wang K, Sun Y, Zhang G, Shen B. Parameters of dynamic contrast-enhanced MRI as imaging markers for angiogenesis and proliferation in human breast cancer. Med Sci Monit. 2015;21:376-82.

9. Mori N, Ota H, Mugikura S, Takasawa C, Ishida T, Watanabe G, Tada H, Watanabe M, Takase K, Takahashi S. Luminal-type breast cancer: correlation of apparent diffusion coefficients with the Ki-67 labeling index. Radiology. 2015;274(1):66-73.

10. Aydin H, Guner B, Esen Bostanci I, Bulut ZM, Aribas BK, Dogan L, Gulcelik MA. Is there any relationship between ADC values of diffusion-weighted imaging and the histopathological prognostic factors of invasive ductal carcinoma? Br J Radiol. 2018:91:20170705.

11. Akın Y, Uğurlu MÜ, Kaya H, Arıbal E. Diagnostic value of diffusion-weighted imaging and apparent diffusion coefficient values in the differentiation of breast lesions, histopathologic subgroups and correlatıon with prognostıc factors using 3.0 Tesla MR. J Breast Health. 2016;12(3):123-32.

12. Arponen $O$, Sudah M, Masarwah A, Taina M, Rautiainen S, Könönen M Sironen R, Kosma VM, Sutela A, Hakumäki J, Vanninen R. Diffusion-weighted imaging in 3.0 Tesla breast MRI: diagnostic performance and tumor characterization using small subregions vs. whole tumor regions of interest. PLoS One. 2015:10(10):e0138702.

13. De Felice C, Cipolla V, Guerrieri D, Santucci D, Musella A, Porfiri LM, Meggiorini ML. Apparent diffusion coefficient on 3.0 Tesla magnetic resonance imaging and prognostic factors in breast cancer. Eur J Gynaecol Oncol. 2014;35(4):408-14.

14. Martincich L, Deantoni V, Bertotto I, Redana S, Kubatzki F, Sarotto I, Rossi V, Liotti M, Ponzone R, Aglietta M, Regge D, Montemurro F. Correlations between diffusion-weighted imaging and breast cancer biomarkers. Eur Radiol. 2012;22(7):1519-28.

15. Choi SY, Chang YW, Park HJ, Kim HJ, Hong SS, Seo DY. Correlation of the apparent diffusion coefficiency values on diffusion-weighted imaging with prognostic factors for breast cancer. Br J Radiol. 2012;85(1016):e474-9.

16. Molinari C, Clauser P, Girometti R, Linda A, Cimino E, Puglisi F, Zuiani C, Bazzocchi M. MR mammography using diffusion-weighted imaging in evaluating breast cancer: a correlation with proliferation index. Radiol Med. 2015;120(10):911-8.

17. Fan M, He T, Zhang P, Zhang J, Li L. Heterogeneity of diffusion-weighted imaging in tumours and the surrounding stroma for prediction of Ki-67 proliferation status in breast cancer. Sci Rep. 2017:7(1):2875.

18. Amornsiripanitch N, Nguyen VT, Rahbar H, Hippe DS, Gadi VK, Rendi MH, Partridge SC. Diffusion-weighted MRI characteristics associated with prognostic pathological factors and recurrence risk in invasive ER+/HER2breast cancers. J Magn Reson Imaging. 2017; https://doi.org/10.1002/jmri. 25909.

19. Shin JK, Kim JY. Dynamic contrast-enhanced and diffusion-weighted MRI of estrogen receptor-positive invasive breast cancers: associations between quantitative MR parameters and Ki-67 proliferation status. J Magn Reson Imaging. 2017:45(1):94-102.

20. Duffy MJ, Harbeck N, Nap M, Molina R, Nicolini A, Senkus E, Cardoso F. Clinical use of biomarkers in breast cancer: updated guidelines from the European Group on Tumor Markers (EGTM). Eur J Cancer. 2017;75:284-98.

21. Li L, Han D, Wang X, Wang Q, Tian J, Yao J, Yuan L, Qian K, Zou Q, Yi W, Zhou E, Yang K. Prognostic values of Ki-67 in neoadjuvant setting for breast cancer: a systematic review and meta-analysis. Future Oncol. 2017:13(11):1021-34.

22. Tao M, Chen S, Zhang X, Zhou Q. Ki-67 labeling index is a predictive marker for a pathological complete response to neoadjuvant chemotherapy in breast cancer: a meta-analysis. Medicine (Baltimore). 2017;96(51):e9384.

23. Kontzoglou K, Palla V, Karaolanis G, Karaiskos I, Alexiou I, Pateras Konstantoudakis K, Stamatakos M. Correlation between Ki67 and breast cancer prognosis. Oncology. 2013;84(4):219-25.

24. Fornasa F. Diffusion-weighted magnetic resonance imaging: what makes water run fast or slow? J Clin Imaging Sci. 2011;1:27.

25. Surov A, Meyer HJ, Wienke A. Correlation between apparent diffusion coefficient (ADC) and cellularity is different in several tumors: a metaanalysis. Oncotarget. 2017:8:59492-9.

26. Surov A, Meyer HJ, Wienke A. Correlation between apparent diffusion coefficient (ADC) and KI 67 in different tumors: a meta-analysis. Part 1: ADC mean. Oncotarget. 2017;8(43):75434-44.

27. He $X$, Chen $Z$, Fu T, Jin $X, Y u T$, Liang $Y$, Jin $X, Y u T$, Liang $Y$, Zhao $X$, Huang L. Ki-67 is a valuable prognostic predictor of lymphoma but its utility varies in lymphoma subtypes: evidence from a systematic meta-analysis. BMC Cancer. 2014;14:153.

28. Schlüter C, Duchrow M, Wohlenberg C, Becker MH, Key G, Flad HD, Gerdes J. The cell proliferation-associated antigen of antibody Ki-67: a very large, ubiquitous nuclear protein with numerous repeated elements, representing a new kind of cell cycle-maintaining proteins. J Cell Biol. 1993;123:513-22.

29. Valentine MT, Perlman ZE, Mitchison TJ, Weitz DA. Mechanical properties of Xenopus egg cytoplasmic extracts. Biophys J. 2005:88:680-9.

30. Zhuang Z, Zhang Q, Zhang D, Cheng F, Suo S, Geng X, Hua J, Xu J. Utility of apparent diffusion coefficient as an imaging biomarker for assessing the proliferative potential of invasive ductal breast cancer. Clin Radiol. 2018;73:473-8.

31. Petrelli F, Viale G, Cabiddu M, Barni S. Prognostic value of different cut-off levels of Ki-67 in breast cancer: a systematic review and meta-analysis of 64,196 patients. Breast Cancer Res Treat. 2015;153(3):477-91.

32. Onishi N, Kanao S, Kataoka M, lima M, Sakaguchi R, Kawai M, Kataoka TR, Mikami Y, Toi M, Togashi K. Apparent diffusion coefficient as a potential surrogate marker for Ki-67 index in mucinous breast carcinoma. J Magn Reson Imaging. 2015;41(3):610-5. 